\title{
Practical Control of Surge Tanks Suffering from Frequent Inlet Flow Upsets
}

Peter Rosander, Alf J. Isaksson, Johan Löfberg, Krister Forsman

Division of Automatic Control

E-mail: rosander@isy.liu.se, alf.isaksson@se.abb.com, johanl@isy.liu.se, krister.forsman@perstorp.com

18th November 2011

Report no.: LiTH-ISY-R-3036

Submitted to IFAC Conference on Advances in PID Control

Address:

Department of Electrical Engineering

Linköpings universitet

SE-581 83 Linköping, Sweden

WWW: http://wWw. control.isy.liu.se

AUTOMATIC CONTROL

REGLERTEKNIK

LINKÖPINGS UNIVERSITET

Technical reports from the Automatic Control group in Linköping are available from http://www. control.isy.liu.se/publications. 


\begin{abstract}
In the presence of frequent inlet flow upsets, tuning of averaging level controllers is typically quite complicated since not only the size of the individual steps but also the time in between the subsequent steps need to considered. One structured way to achieve optimal filtering for such a case is to use Robust Model Predictive Control. The robust MPC controller is, however, quite computationally demanding and not easy to implement. In this paper two linear controllers, which mimic the behavior of the robust MPC are proposed. Tuning guidelines to avoid violation of the tank level constraints as well as to achieve optimal filtering are presented.
\end{abstract}

Keywords: Averaging level control, PI controllers, Surge tanks 


\title{
Practical Control of Surge Tanks Suffering from Frequent Inlet Flow Upsets
}

\author{
Peter Rosander, * Alf J. Isaksson, * Johan Löfberg, * \\ Krister Forsman** \\ * Department of Electrical Engineering, Linköping University, \\ SE-581-83 Sweden (email: \{rosander,alf,johanl\}@isy.liu.se) \\ ** Perstorp AB, (email: krister.forsman@perstorp.com)
}

\begin{abstract}
In the presence of frequent inlet flow upsets, tuning of averaging level controllers is typically quite complicated since not only the size of the individual steps but also the time in between the subsequent steps need to considered. One structured way to achieve optimal filtering for such a case is to use Robust Model Predictive Control. The robust MPC controller is, however, quite computationally demanding and not easy to implement. In this paper two linear controllers, which mimic the behavior of the robust MPC are proposed. Tuning guidelines to avoid violation of the tank level constraints as well as to achieve optimal filtering are presented.
\end{abstract}

Keywords: Averaging level control, PI controllers, surge tanks

\section{INTRODUCTION}

The objective of averaging level controllers is to keep the outlet flow of the surge tank smooth by using the tank capacity to average out the effect of inlet flow upsets. Following an inlet flow upset, averaging is typically obtained by initially allowing the tank level to deviate from its nominal set-point while slowly adapting the outlet to the new throughput and ultimately bringing back the tank level to the set-point.

Early approaches to averaging control are the PL controller in (Luyben and Buckley, 1977) and the nonlinear approaches by Shunta and Fehervari (1976). The (de-)tuning of PI controllers has been addressed in (Cheung and Luyben, 1979), (Kelly, 1998) and (Shin et al., 2008) amongst others. A control structure permitting separate responses to set-point and load disturbances were proposed by $\mathrm{Wu}$ et al. (2001). The optimal non-linear continuous- and discrete-time controllers were derived in (McDonald et al., 1986) and in (Campo and Morari, 1989) respectively.

We have encountered an application in of Perstorp's factories where the previously mentioned approaches struggle to obtain satisfactory flow filtering. The reason for this is that traditionally inlet flow upsets have been treated as separate occurrences, but, as seen in Figure 1, this clearly does not hold in this case. This complicates the tuning since not only the size but also the time in between upsets need to be considered. In (Rosander et al., 2011) optimal filtering in the presence of frequent inlet flow upsets was obtained using robust Model Predictive Control (MPC). The robust MPC controller differs from previous approaches in that it does not return the tank level to a fixed set-point following an inlet flow step. Instead the steady state tank level depends on the current inlet flow

* This work was funded by the Swedish Foundation for Strategic Research as part of Process Industry Center Linköping (PIC-LI). level. In (Rosander et al., 2012) it is shown that the robust MPC controller achieves comparable performance as the optimal level controller by (McDonald et al., 1986), even when upset are infrequent.

The robust MPC or the non-linear controller derived in (Rosander et al., 2012) are, however, quite complex which limit their industrial applicability. In this paper we propose and analyze two linear controllers which mimic the behavior of the robust MPC controller. The first one is a special version of the proportional controller while the other one is a PI whose set-point is not fixed but given as an affine map of the inlet flow.

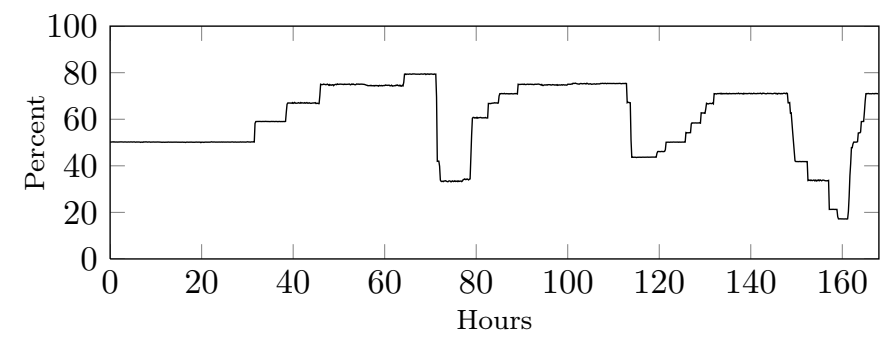

Fig. 1. Inlet flow data from Perstorp AB last week of March 2011.

\section{SYSTEM DESCRIPTION}

We consider a cylindrical buffer tank with level $y$ and liquid constant density inlet and outlet flows $q_{\text {in }}$ and $q_{\text {out }}$ respectively. For the sake of notational brevity it is assumed that the outlet flow can be directly manipulated, $u=q_{\text {out }}$. Using mass balance we obtain the continuous time model

$$
\dot{y}(t)=k_{v}\left(q_{i n}(t)-u(t)\right),
$$

where $k_{v}$ is the cross-sectional area inverted. Furthermore we assume that all quantities are given in percent and that the outlet and inlet flow have equal range 


$$
\begin{aligned}
& y_{\min } \leq y \leq y_{\max } \\
& q_{\min } \leq q_{\text {in }}, u \leq q_{\max }
\end{aligned}
$$

Here $q_{\min }=y_{\min }=0 \%$ and $q_{\max }=y_{\max }=100 \%$ if no extra safety limitations are put on the tank level. The assumption (2b) guarantees that we do not risk violating the tank level constraints due to lack of outlet flow capacity. Consequently we could allow the outlet flow to have greater range than the inlet flow, but for the purpose of this paper that would mainly complicate the notation. For the same reason we assume that the inlet flow is directly measurable. In any case, the linear dynamics of the system allow for a straightforward estimation of the inlet flow using the Kalman filter as shown in (Khanbaghi et al., 2001).

Good averaging level control is characterized by a smooth outlet flow, i.e., that $\dot{u}$ is "small". Typically this is quantified by either the Euclidean norm

$$
\|\dot{u}\|_{2}^{2}=\int_{0}^{\infty} \dot{u}(t)^{2} d t
$$

or the maximum change of the outlet flow

$$
\|\dot{u}\|_{\infty}=\max _{t}|\dot{u}(t)| \text {. }
$$

Which criterion that best captures "good flow filtering" depends of course on the nature of the downstream processes. In this paper we will display both to give as complete a picture as possible.

\section{ROBUST AVERAGING LEVEL CONTROL}

We will briefly describe the robust MPC approach to averaging level control taken in (Rosander et al., 2011).

By first discretizing the model (1), optimal flow filtering while directly accounting for future inlet flow upsets is achieved by, in every sample instant, solving the optimization problem

$$
\begin{aligned}
& \min _{u(0: N)} \max _{q_{i n}(1: N)}\|u(k)-u(k-1)\|_{\infty} \\
& \text { subject to } \\
& y(k+1)=y(k)+T_{s} k_{v}\left(q_{i n}(k)-u(k)\right) \\
& y(0), q_{i n}(0), u(-1) \text { known } \\
& y(1: N) \in\left[y_{\min }, y_{\max }\right] \forall q_{i n}(1: N) \in\left[q_{\min }, q_{\max }\right] \\
& q_{\text {in }}(1: N), u(1: N) \in\left[q_{\min }, q_{\max }\right]
\end{aligned}
$$

where $y(0)$ and $q_{i n}(0)$ are the current tank level and inlet flow respectively and $u(-1)$ is the actuated control signal from the previous sampling instant. Note that the only requirement put on the tank level is that it must stay within bounds $(5 \mathrm{~d})$. The controller is thus allowed to "decide" the optimal tank level.

The behavior of the robust MPC controller differs from most previous approaches (apart from the $\mathrm{P}$ controller) as it does not return the tank level to a fixed set-point, but instead the steady state tank level depends on the actual level of the inlet flow. This is illustrated in Figure 2 for a sample feed where both the robust MPC controller as well as a standard PI controller is used to control the tank. The nature of the mapping from inlet flow to steady state tank level depends on the prediction horizon, but for prediction horizons that capture the transient response of the system, the mapping stays almost constant, see
Figure 3. In Figure 3 also the approximation, the affine mapping, used in this paper is shown.

Inlet and Outlet Flows

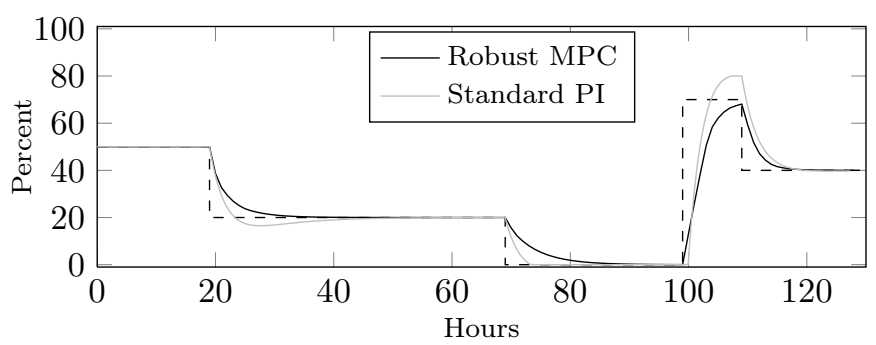

Tank Level

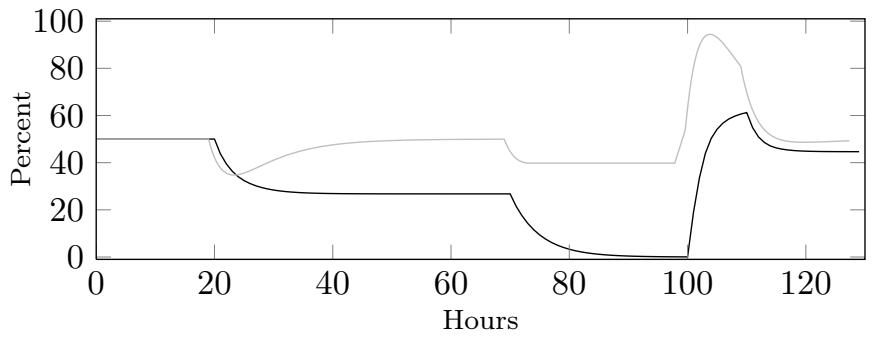

Fig. 2. Simulation of closed loop robust MPC with $N=$ 20, shown in solid black. For comparison purposes a standard PI-controller with $K_{c}=-1.5$ and $T_{I}=10$ is also shown in gray.

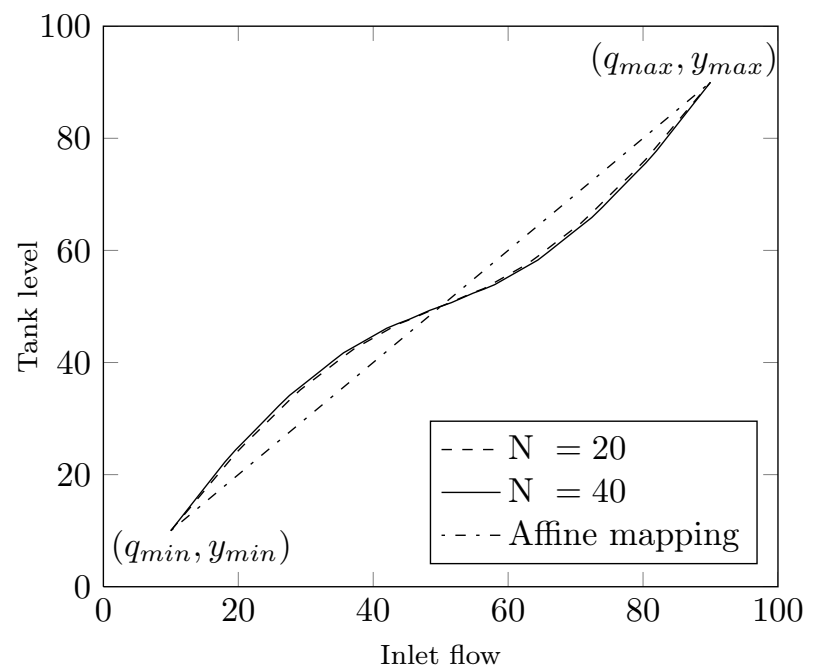

Fig. 3. The steady state tank level as a function of the inlet flow for different prediction horizons and $k_{v}=\frac{1}{3}$. Shown is also the affine mapping that will be used in this paper.

\subsection{Robust Optimal Averaging Level Control}

The robust MPC controller does not permit an analytical analysis, and to still obtain an explicit estimate of its performance we do the following. The filtering problem is formulated as an optimal control problem for which robustness towards future inlet flow upsets is obtained by letting the steady state tank level be given by the affine map shown in Figure 3. A robustly optimal controller can thus be obtained by solving the optimization problem 


$$
\begin{aligned}
& \min \|\dot{u}\|_{\infty} \text { or }\|\dot{u}\|_{2}^{2} \\
& \text { subject to } \\
& \dot{y}=k_{v}\left(q_{i n}(t)-u(t)\right) \\
& q_{\text {in }}(t)= \begin{cases}q_{0}, & t<0 \\
q_{0}+A, & t \geq 0\end{cases} \\
& y(0)=K_{S P} q_{0}+b_{S P} \\
& y(T)=K_{S P}\left(q_{0}+A\right)+b_{S P}, T \text { free }
\end{aligned}
$$

where $A$ is the size of the inlet flow upset. The constraints (6d) and (6e) ensure that the resulting controller will display the same level-adaption as the robust MPC controller. Using Figure 3 the parameters of this mapping can be found to be

$$
\begin{aligned}
K_{S P} & =\frac{y_{\max }-y_{\min }}{q_{\max }-q_{\min }}, \\
b_{S P} & =\frac{q_{\max } y_{\min }-q_{\min } y_{\max }}{q_{\max }-q_{\min }} .
\end{aligned}
$$

The formulation (6) assumes that the system is in steady state but that can be relaxed.

The solution to (6) for the infinity norm was presented in (Rosander et al., 2012), where it was also shown that the performance of the robustly optimal averaging level controller was comparable to that of the truly (non-robust) optimal averaging controller by McDonald et al. (1986). The Euclidean norm case was derived in (Rosander, 2011). Summarizing the results, the performance criteria for the robustly optimal controller evaluates to

$$
\begin{aligned}
J_{O P T, 2} & =\frac{4 k_{v} A^{2}}{9 K_{S P}}, \\
J_{O P T, \infty} & =\frac{k_{v}|A|}{2 K_{S P}} .
\end{aligned}
$$

This gives us a lower bound with which we can compare the performance achieved by the linear controllers.

Both optimal controllers as well as the robust MPC controller are, however, rather complicated (from a process control perspective). Especially since surge tanks are amongst the simpler processes in a plant. Instead we use the insights gained to propose simpler linear feedback controllers, which as it turns out actually can achieve comparable filtering performance.

\section{OPTIMAL FILTERING P CONTROL}

The obvious choice of a linear controller which gives an offset in the steady tank level, as in Figure 2, is the P controller. $\mathrm{P}$ control of surge tanks is admittedly nothing new and has previously been advocated by (Rivera et al. 1986) and (Taylor and la Grange, 2002) amongst others.

\section{A $\mathrm{P}$ controller is given by}

$$
u(t)=K_{P}(r-y(t))+b_{P}=-K_{P} y(t)+\underbrace{K_{P} r+b_{P}}_{\text {bias }},
$$

and thus the parameter $r$ is not really a set-point but just another bias term.

Inserting (1) into (9) we obtain that for an inlet flow step of size $A$

$$
u(t)=A\left(1-e^{K_{P} k_{v} t}\right) .
$$

Using this, straightforward, but tedious, calculations give that the performance of the $\mathrm{P}$ controller evaluates to

$$
\begin{aligned}
J_{P, 2} & =\|\dot{u}\|_{2}^{2}=-\frac{A^{2} k_{v} K_{P}}{2}, \\
J_{P, \infty} & =\|\dot{u}\|_{\infty}=-|A| k_{v} K_{P},
\end{aligned}
$$

where the minus signs come from the fact that $K_{P}<0$ to obtain stability. Not surprisingly, we see that a small $\left|K_{P}\right|$ is preferable from a filtering perspective. The smallest $\left|K_{P}\right|$ that will fulfill the constraints (2) can be found by mapping minimum and maximum tank level to minimum and maximum inlet flow

$$
\begin{aligned}
K_{P} & =-\frac{q_{\max }-q_{\min }}{y_{\max }-y_{\min }}, \\
\text { bias } & =\frac{q_{\min } y_{\max }-q_{\max } y_{\min }}{y_{\max }-y_{\min }} .
\end{aligned}
$$

This means that $K_{S P}=-\frac{1}{K_{P}}$ and we can thus conclude that in terms of the two-norm the performance degrades by approximately $12 \%$ while the infinity-norm actually is twice as bad.

Apart from really bad flow smoothing in terms of the infinity-norm criterion there is also an issue with how the controller is perceived by the operators. The term $b_{P}$ is typically not available since it is used to handle bump less transfers between operating modes. To then obtain the bias as in (12) a rather unintuitive set-point must be used, and furthermore one that is never attained by the controller. The risk is that the controller is perceived as not functioning correctly and hence disconnected.

A PI controller, put in cascade mode, whose set-point is given by a calculation block resolves these two drawbacks of the P controller. The operators can easily see what setpoint the system is trying to achieve and the set-point is also obtained. The PI controller also achieves better smoothing, especially in terms of worst case performance.

\section{PI WITH VARIABLE SET-POINT}

One easy way to mimic the robust MPC with a PI controller is to let its set-point be given by the affine mapping

$$
r(t)=K_{S P} q_{i n}(t)+b_{S P}
$$

where $K_{S P}$ and $b_{S P}$ are given by $(7 \mathrm{a})$ and $(7 \mathrm{~b})$ respectively. A block diagram of the proposed control structure is shown in Figure 4 where the block $M A P$ is the affine

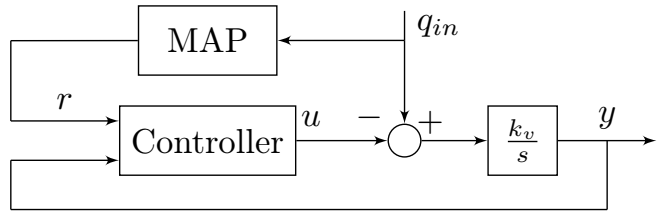

Fig. 4. Block diagram of the proposed PI controller.

map (13). In the subsequent analysis we assume that the effect $b_{S P}$ has on $u$ and $y$ has reached its steady state value and therefore only focus on $q_{i n}$.

\subsection{Transfer functions}

In addition to changing the set-point according to (13) a $\beta$-factor in the controller will be used

$$
U(s)=K_{c}\left(\beta R(s)-Y(s)+\frac{1}{T_{I} s}(R(s)-Y(s))\right) .
$$


The resulting closed loop dynamics from $Q_{i n}$ can be derived to be

$$
\begin{aligned}
& Y(s)=\frac{k_{v}\left(T_{I} s-K_{c} \beta K_{S P} T_{I} s-K_{c} K_{S P}\right)}{T_{I} s^{2}-k_{v} K_{c} T_{I} s-k_{v} K_{c}} Q_{i n}(s), \\
& U(s)=\frac{K_{c}\left(\beta K_{S P} T_{I} s^{2}+\left(K_{S P}-k_{v} T_{I}\right) s+1\right)}{T_{I} s^{2}-k_{v} K_{c} T_{I} s-k_{v} K_{c}} Q_{i n}(s) .
\end{aligned}
$$

\section{TUNING OF PI}

When adapting the level, additional requirements (apart from closed loop stability) have to be put on the controller: an inlet flow step response cannot yield an under or overshoot. Why this is the case can be understood by viewing an example: Assume that the system is in steady state at $t=t^{\prime}$. Then $q_{i n}$ performs a step from $q_{\text {min }}$ to $q_{\max }$ and eventually let us say at $t=t^{\prime \prime}$ the system will again be in steady state. We then have that $u\left(t^{\prime}\right)=q_{\text {min }}$, $y\left(t^{\prime}\right)=y_{\min }, u\left(t^{\prime \prime}\right)=q_{\max }$ and $y\left(t^{\prime \prime}\right)=y_{\max }$. An overshoot in either $y(t)$ or $u(t)^{1}$ would then lead to tank overflow while an undershoot would give an empty tank.

Before turning to investigate the under and overshoot of the system we note that the controller has a direct term from the inlet to the outlet flow

$$
\beta K_{c} K_{S P}
$$

A step in the inlet flow will thus give a discontinuous outlet flow which obviously contradicts the main idea of using surge tanks. The obvious choice is thus $\beta=0$ to decouple the outlet from the inlet flow. This yields the transfer functions

$$
\begin{aligned}
Y(s) & =\frac{k_{v}\left(K_{S P} T_{I} s-K_{c} K_{S P}\right)}{T_{I} s^{2}-k_{v} K_{c} T_{I} s-k_{v} K_{c}} Q_{i n}(s), \\
U(s) & =\frac{\left.K_{c}\left(\left(K_{S P}-k_{v} T_{I}\right) s+k_{v}\right)\right)}{T_{I} s^{2}-k_{v} K_{c} T_{I} s-k_{v} K_{c}} Q_{i n}(s) .
\end{aligned}
$$

Both systems are thus of the form $K \frac{s+z}{\left(s+p_{1}\right)\left(s+p_{2}\right)}$ for which there exist necessary and sufficient conditions to give a non under or overshooting step response. The requirements, as derived in (Kwon et al., 2002), are real stable poles and that the zero is farther away from the origin than the rightmost pole

$$
-\Re\left\{p_{1}, p_{2}\right\}<0, \Im\left\{p_{1}, p_{2}\right\}=0, z \geq \max \Re\left\{p_{1}, p_{2}\right\} .
$$

The resulting step response will actually be monotone and henceforth we will refer to monotonicity as the sought after behavior.

As derived in Appendix A the system given by (17a) and (17b) has a monotone step response if and only if

$$
\begin{aligned}
& T_{I}>\frac{K_{S P}}{k_{v}}, \\
& K_{c} \leq-\frac{4}{k_{v} T_{I}}, T_{I} \in\left(\frac{K_{S P}}{k_{v}}, \frac{2 K_{S P}}{k_{v}}\right], \\
& K_{c} \leq \frac{k_{v} T_{I}}{K_{S P}\left(K_{S P}-k_{v} T_{I}\right)}, T_{I}>\frac{2 K_{S P}}{k_{v}} .
\end{aligned}
$$

The permitted area, defined by (19), is shown in Figure 5 for some numerical values of $k_{v}$ and $K_{S P}$. In general $k_{v}$ is, of course, not known exactly but replacing it by

\footnotetext{
1 Strictly speaking this is only required close to the boundary and might thus be obtained for a non-linear gain. Relaxing (2b) would also permit over or undershoot in $u(t)$ even in the linear case.
}

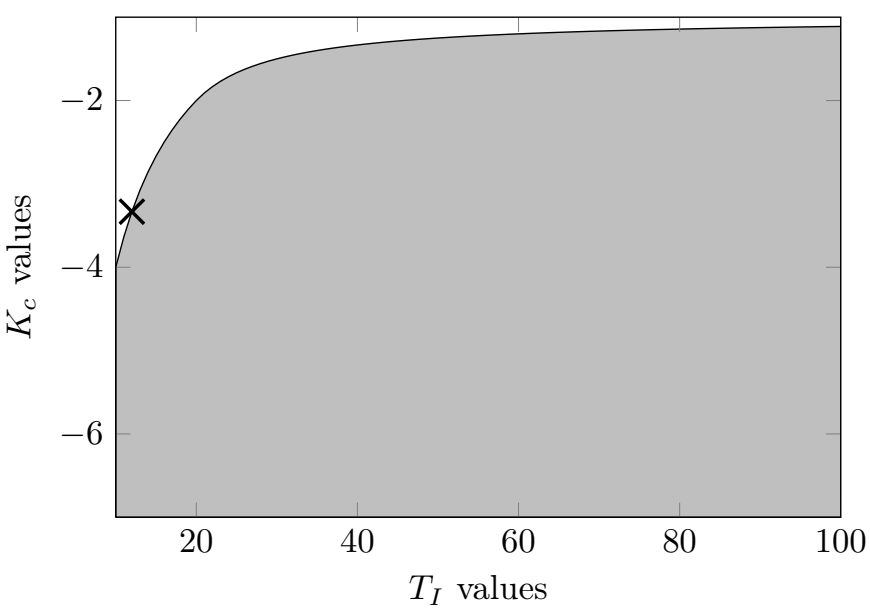

Fig. 5. Shaded area is the permitted region for $k_{v}=\frac{1}{10}, K_{S P}=1$ and $b_{S P}=0$. The optimal tuning for these system parameters is also shown as a cross.

appropriate estimates (for example a lower bound in (19a) and an upper one in (19b)) it is still possible to ensure a monotone step response in $y$ and $u$.

\subsection{Optimal performance for a step disturbance}

Provided that the parameters fulfill (19) how should they be chosen to achieve optimal filtering? We derive the optimal parameters for the Euclidean norm since it yields an analytical expression. Under the assumption of a step disturbance of size $A$ we thus want to minimize

$$
\begin{aligned}
& J_{P I, 2}=\|\dot{u}\|_{2}^{2}=\int_{0}^{\infty} \dot{u}^{2}(t) d t \\
& \text { subject to }
\end{aligned}
$$

(17b), (19)

The calculation of $J_{P I, 2}$ has to be split into three parts depending on the pole-zero configuration of the system. For $K_{c}=-\frac{4}{k_{v} T_{I}}$ the system has a double pole and obtains

$$
J_{P I, 2}^{\text {double }}=\frac{5 k_{v}^{2} T_{I}^{2}+4 K_{S P}^{2}-8 k_{v} T_{I} K_{S P}}{2 k_{v}^{2} T_{I}^{3}}
$$

where the superscript double is used to denote a double pole. For $K_{c}=\frac{k_{v} T_{I}}{K_{S P}\left(K_{S P}-k_{v} T_{I}\right)}$ pole zero cancellation occurs (regardless of $T_{I}$ value) and the PI controller essentially $^{2}$ becomes a $\mathrm{P}$ controller and consequently the criterion evaluates to

$$
J_{P I, 2}^{\text {single }}=J_{P, 2}=\frac{k_{v}}{2 K_{S P}} .
$$

For $K_{c}$ not on the boundary we have an over damped second order system with performance criterion

$$
J_{P I, 2}^{2}=\frac{\left(2 k_{v} T_{I} K_{S P}-K_{S P}^{2}-k_{v}^{2} T_{I}^{2}\right) K_{c}+k_{v} T_{I}}{2 k_{v} T_{I}^{2}} .
$$

Investigating $J_{P I, 2}^{2}$ closer we note that

$$
\begin{aligned}
\frac{\partial J_{P I, 2}^{2}}{\partial K_{c}}= & 2 k_{v} T_{I} K_{S P}-K_{S P}^{2}-k_{v}^{2} T_{I}^{2}= \\
& -\left(k_{v} T_{I}-K_{S P}\right)^{2}<0
\end{aligned}
$$

and it is thus optimal to choose $K_{c}$ on the boundary. Consequently either $J_{P I, 2}^{\text {double }}$ or $J_{P I, 2}^{\text {single }}$ is the optimal objective

\footnotetext{
2 The integrating part of the controller is of course still present.
} 
function. The optimal $T_{I}$-value for $(21)$ can be derived by solving

$$
\frac{d J_{P I, 2}^{\text {double }}}{d T_{I}}=0 \quad \frac{d^{2} J_{P I, 2}^{\text {double }}}{d T_{I}^{2}}>0,
$$

yielding

$$
T_{I}=\frac{6 K_{S P}}{5 k_{v}}
$$

Inserting this into (21) the objective evaluates to

$$
J_{P I, 2}^{\text {double }}=\frac{25 k_{v}}{54 K_{S P}}
$$

which clearly is smaller than $J_{P I, 2}^{\text {single }}$. Concluding, the optimal tuning is

$$
\begin{aligned}
K_{c} & =-\frac{4}{k_{v} T_{I}}, \\
T_{I} & =\frac{6 K_{S P}}{5 k_{v}} .
\end{aligned}
$$

Turning to the maximum criterion we obtain that with the proposed tuning it evaluates to

$$
J_{P I, \infty}=\frac{10|A| k_{v} e^{-\frac{1}{2}}}{9 K_{S P}} .
$$

Comparing the performance values obtained for the proposed PI controller with that of the robustly optimal controllers we see that it is $4 \%$ and $35 \%^{3}$ worse in terms of Euclidean and infinity norm respectively. Furthermore, the PI is approximately $7 \%$ and $33 \%$ better than the $\mathrm{P}$ controller in terms of the two and infinity norm criterion respectively.

\section{SIMULATION RESULTS}

To illustrate the benefits with the proposed controllers they are compared with a standard PI with a fixed setpoint (hereafter denoted Fix-PI) on data for the first 21 weeks ( 5 months) of 2011. The system is assumed to have $k_{v}=\frac{1}{3}$ and

$$
\begin{aligned}
& q_{\min }=y_{\min }=0, \\
& q_{\max }=y_{\max }=100 .
\end{aligned}
$$

The Fix-PI is augmented with the tracking anti-windup (tracking gain $\frac{1}{T_{a}}=\frac{1}{T_{I}}$ ) in (Tharayil and Alleyne, 2002). Furthermore the minimum and maximum outlet flows are perturbed an additional $5 \%$ to improve the Fix-PI's handling of flows on or close to the boundary, hence an outlet flow of $-5 \%$ and $105 \%$ is permitted for the Fix-PI controller. The tuning of the Fix-PI is a delicate business as a too detuned controller will violate the level constraints and too tight tuning will give bad disturbance attenuation. We will do what is typically done in industry: use historical data to find parameters that are optimal for that data and that are somewhat robust towards the uncertain future. We use four months of data from 2010 and find a tuning which is optimal for that data. Robustness towards future uncertainty is obtained by requiring that the control parameters must keep the tank level within $10 \%$ and $90 \%$ for the 2010 data. This yields the parameters

$$
\begin{aligned}
K_{c}^{2010} & =-1.1, \\
T_{I}^{2010} & =3.5 .
\end{aligned}
$$

3 This could have been decreased even further by instead minimizing $\|\dot{u}\|_{\infty}$.
Euclidean Norm

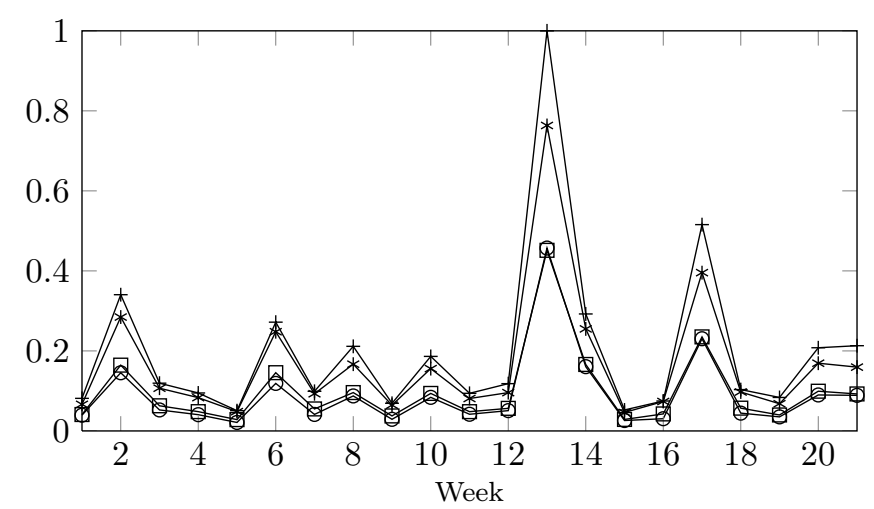

Infinity Norm

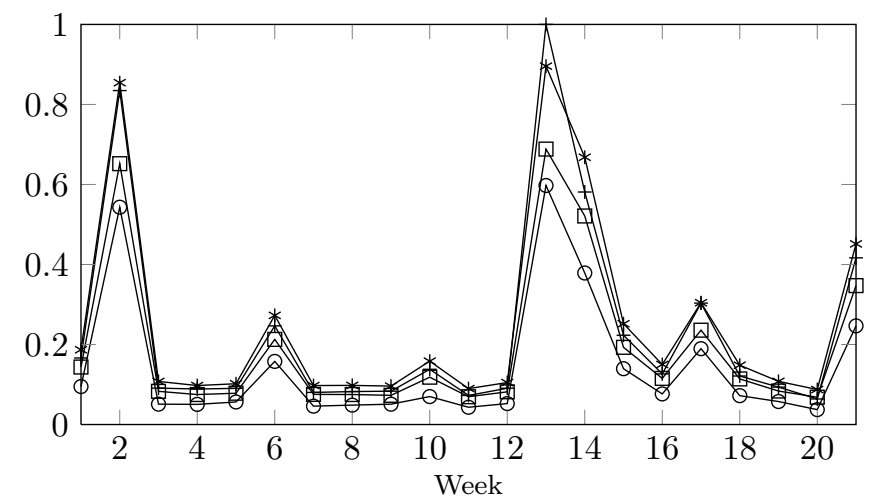

Fig. 6. Normalized Euclidean and infinity norm for the controllers: Fix-PI controller with the tuning using data from $2010(+)$, the Fix-PI controller with the oracle tuning using the actual data from 2011 (*), the optimal filtering $\mathrm{P}$ controller $(\square)$ and the variable setpoint PI controller (o).

To obtain a lower bound for what is possible to achieve with a Fix-PI we use the actual data from 2011 and by an extensive search the optimal $K_{c}$ and $T_{I}$ values are found. The parameters which obtained the least average Euclidean norm value were

$$
\begin{aligned}
K_{c}^{\text {oracle }} & =-1.3, \\
T_{I}^{\text {oracle }} & =9.5 .
\end{aligned}
$$

The two tunings of Fix-PI as well as the proposed $\mathrm{P}$ and PI controllers were simulated for the 2011 data and their weekly performance is showed in Figure 6 . The $\mathrm{P}$ controller performs, as expected, almost as well as the variable setpoint PI controller in terms of the Euclidean norm, but much worse in terms of the infinity norm. Furthermore we see that the oracle tuning of the Fix-PI (which is a lower bound for what a PI can achieve) is still $\approx 50 \%$ as bad as the variable set-point PI controller. The tuning using data from 2010 performs almost as well as the oracle solution.

\section{CONCLUSIONS}

Two linear controllers that mimics the behavior of the robust MPC have been presented. Tuning guidelines to guarantee that the level does not violate any constraints were derived. The PI with a variable set-point was shown to perform considerable better than the $\mathrm{P}$ controller in terms of worst case performance. 
Future work includes investigating if the inlet flow characteristics can be used to design alternatives to the affine mapping and if gain-scheduling can be used to just enforce monotonic step responses close to the boundary. The addition of a low pass filter to the mapping should probably also be added to improve the controller's noise sensitivity.

Another extension is to apply the PI controller to a cascade of surge tanks. The inlet flow to the second tank is then not well described as a step as it is the outlet from the first tank. The optimal control parameters might then differ and whether the tuning can be done sequentially or not needs to be investigated.

\section{REFERENCES}

Campo, P. and Morari, M. (1989). Model predictive optimal averaging level control. AIChE Journal, 35(4), 579-591.

Cheung, T. and Luyben, W. (1979). Liquid-level control in single tanks and cascade of tanks with proportional-only and proportional-integral feedback control. Industrial and Engineering Chemistry Fundamentals, 18(1), 15-21.

Kelly, J. (1998). Tuning digital PI controllers for minimal variance in manipulated input moves applied to imbalanced systems with delay. Canadian Journal of Chemical Engineering, 76(5), 967-974.

Khanbaghi, M., Allison, B., and Harper, R. (2001). Optimal averaging level control for surge tanks. In IEEE Conference on Advanced Process Control Applications for Industry. Vancouver, Canada.

Kwon, B.M., Lee, M.E., and Kwon, O.K. (2002). On nonovershooting of monotone nondecreasing step response of second-order systems. Transactions on Control, Automation and Systems Engingeering, 4(4), 283288.

Luyben, W. and Buckley, P. (1977). A proportional-lag level controller. Instrumentation Technology, 18(1), 6568.

McDonald, K., McAvoy, T., and Tits, A. (1986). Optimal averaging level control. AIChE Journal, 32(1), 75-86.

Rivera, D., Morari, M., and Skogestad, S. (1986). Internal model control .4. PID controller-design. Industrial 8 Engineering Chemistry Process Design and Development, 25(1), 252-265.

Rosander, P. (2011). Euclidean norm robust optimal averaging level control. Technical Report LiTH-ISY-R3034, Department of Electrical Engineering, Linköping University, SE-581 83 Linköping, Sweden.

Rosander, P., Isaksson, A., Löfberg, J., and Forsman, K. (2011). Robust averaging level control. In Proccedings of the 2011 AIChE Annual Meeting. Minneapolis,USA.

Rosander, P., Isaksson, A., Löfberg, J., and Forsman, K. (2012). Performace analysis of robust averaging level control. Accepted for Chemical Process Control VIII.

Shin, J., Lee, J., Park, S., Koo, K., and Lee, M. (2008). Analytical design of a proportional-integral controller for constrained optimal regulatory control of inventory loop. Control Engineering Practice, 16(11), 1391-1397.

Shunta, J. and Fehervari, W. (1976). Nonlinear control of liquid-level. Instrumentation Technology, 23(1), 43-48.

Taylor, A. and la Grange, T. (2002). Optimize surge vessel control - proportional only algorithm complements multivariable predictive control. Hydrocarbon Processing, $81(5), 49-52$.
Tharayil, M. and Alleyne, A. (2002). A generalized PID error governing scheme for SMART/SBLI control. In Proceedings of the American Control Conference, 2002. Wu, K., Yu, C., and Cheng, Y. (2001). A two degree of freedom level control. Journal of Process Control, 11(3), 311-319.

\section{Appendix A. MONOTONE STEP RESPONSE}

With $\beta=0$ the system has poles

$$
\left\{-p_{1},-p_{2}\right\}=\frac{k_{v} K_{c} T_{I} \pm \sqrt{k_{v}^{2} K_{c}^{2} T_{I}^{2}+4 k_{v} K_{c} T_{I}}}{2 T_{I}},
$$

where $-p_{1}$ is the rightmost one. The zeros are placed in

$$
-z_{q_{i n}, y}=\frac{K_{c} K_{S P}}{T_{I}},-z_{q_{i n}, u}=\frac{k_{v}}{K_{S P}-k_{v} T_{I}} .
$$

Stable poles are obtained if $K_{c}<0$ and real poles iff

$$
k_{v}^{2} K_{c}^{2} T_{I}^{2}+4 k_{v} K_{c} T_{I} \geq 0 \Leftrightarrow K_{c} \leq-\frac{4}{k_{v} T_{I}} .
$$

Straightforward calculations gives that $-z_{q_{i n}, y} \leq-p_{1}$ is fulfilled iff

$$
\begin{array}{r}
K_{c} \leq \frac{k_{v} T_{I}}{K_{S P}\left(K_{S P}-k_{v} T_{I}\right)}, T_{I}>\frac{2 K_{S P}}{k_{v}} \\
K_{c} \leq-\frac{4}{k_{v} T_{I}}, T_{I} \leq \frac{2 K_{S P}}{k_{v}} .
\end{array}
$$

The condition $-z_{q_{i n}, u} \leq-p_{1}$ requires more attention. A necessary condition is that the zero lies in the left half plane

$$
-z_{q_{i n}, u}<0 \Leftrightarrow T_{I}>\frac{K_{S P}}{k_{v}} .
$$

That fulfilled it follows that

$$
\begin{aligned}
& \frac{k_{v}}{K_{S P}-k_{v} T_{I}} \leq \frac{k_{v} K_{c} T_{I}+\sqrt{k_{v}^{2} T_{I}^{2} K_{c}^{2}+4 k_{v} T_{I} K_{c}}}{2 T_{I}} \Leftrightarrow \\
& \frac{2 k_{v} T_{I}-\alpha\left(k_{v} K_{c} T_{I}+\sqrt{k_{v}^{2} T_{I}^{2} K_{c}^{2}+4 k_{v} T_{I} K_{c}}\right)}{2 \alpha T_{I}} \leq 0,
\end{aligned}
$$

where $\alpha \triangleq K_{S P}-k_{v} T_{I}$. Since $\alpha<0$ it follows that

$$
\underbrace{-\alpha \sqrt{k_{v}^{2} T_{I}^{2} K_{c}^{2}+4 k_{v} T_{I} K_{c}}}_{\geq 0} \geq k_{v} T_{I}\left(\alpha K_{c}-2\right) .
$$

We note that for a negative right-hand side

$$
K_{c} \geq \frac{2}{K_{S P}-k_{v} T_{I}}
$$

the inequality is trivially fulfilled and if not we can square both to obtain the requirement

$$
K_{c} \leq \frac{k_{v} T_{I}}{K_{S P}\left(K_{S P}-k_{v} T_{I}\right)} .
$$

It turns out that (A.8) and (A.9) do not impose any additional restrictions on $K_{c}$ since

$$
\frac{-4}{k_{v} T_{I}} \leq \frac{k_{v} T_{I}}{K_{S P}\left(K_{S P}-k_{v} T_{I}\right)} \Leftrightarrow\left(k_{v} T_{I}-2 K_{S P}\right)^{2} \geq 0 .
$$

Combining (A.4) and (A.5) concludes the derivation. 


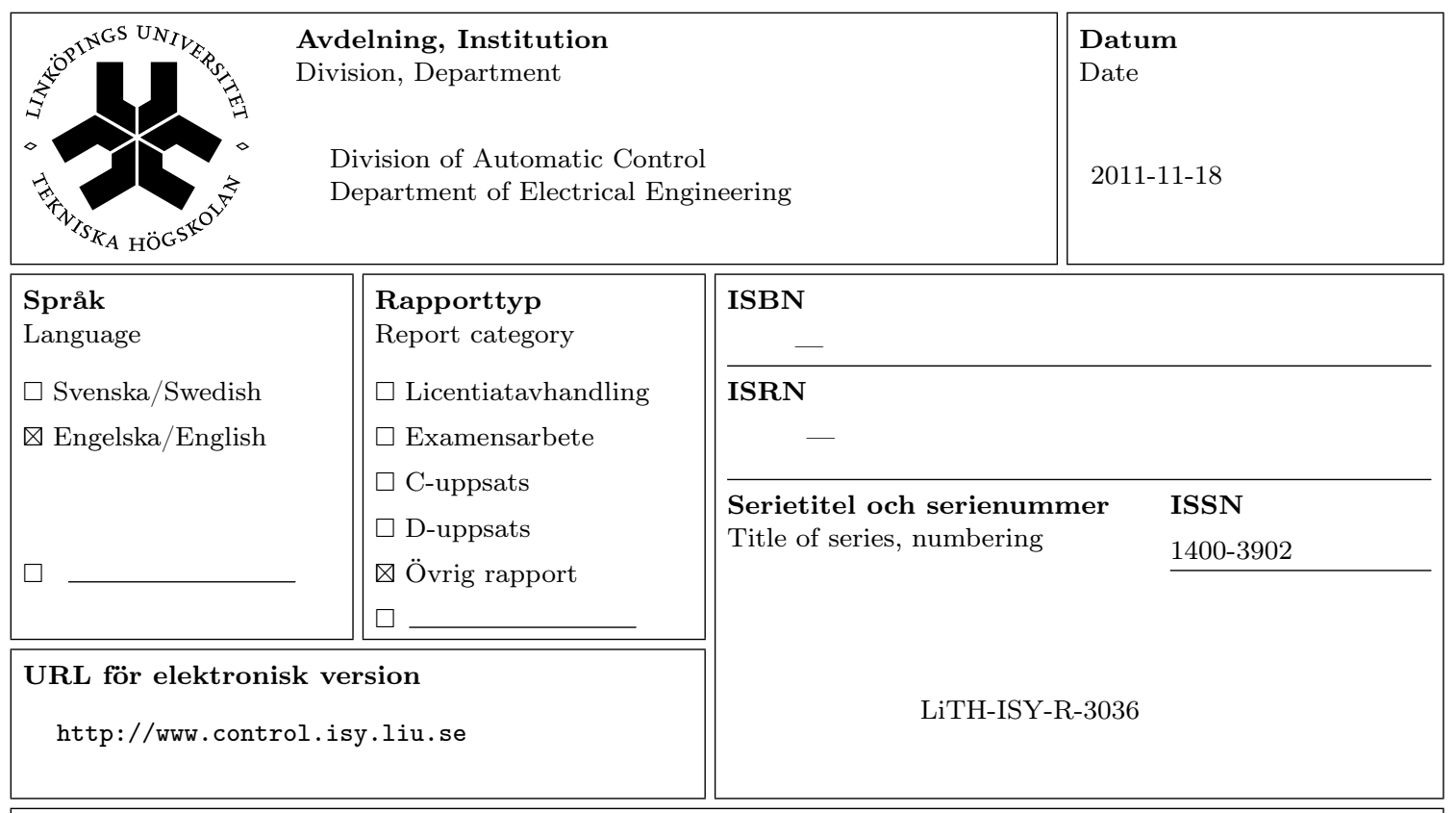

Titel Practical Control of Surge Tanks Suffering from Frequent Inlet Flow Upsets

Title

Författare Peter Rosander, Alf J. Isaksson, Johan Löfberg, Krister Forsman

Author

\section{Sammanfattning}

Abstract

In the presence of frequent inlet flow upsets, tuning of averaging level controllers is typically quite complicated since not only the size of the individual steps but also the time in between the subsequent steps need to considered. One structured way to achieve optimal filtering for such a case is to use Robust Model Predictive Control. The robust MPC controller is, however, quite computationally demanding and not easy to implement. In this paper two linear controllers, which mimic the behavior of the robust MPC are proposed. Tuning guidelines to avoid violation of the tank level constraints as well as to achieve optimal filtering are presented. 